\title{
Challenges in lung and thoracic pathology
}

\author{
Sanja Dacic ${ }^{1} \cdot$ Philipp Ströbel ${ }^{2}$
}

Published online: 5 March 2021

(C) The Author(s), under exclusive licence to Springer-Verlag GmbH, DE part of Springer Nature 2021

Thoracic pathology is a highly subspecialized and challenging area of diagnostic surgical pathology with significant recent updates in the classification of neoplastic and non-neoplastic diseases. Advances in our understanding of the genomics of thoracic tumors are reshaping histologic classifications to make them more clinically relevant. Although molecular biology has greatly helped to better understand known diseases and define new entities, international collaborations and exchange of expertise and sample collections have become more important than ever. If anything, the current COVID19 pandemic with its urgent necessity to learn as much as possible and as soon as possible about a severe lung disease that most of us had never encountered before has dramatically shown the need for (and the power of) coordinated efforts of the global research community.

Metovic and colleagues start by describing the histologic and molecular classification of neuroendocrine tumors of the lung. They provide a detailed approach to the histologic and immunohistochemical diagnosis of carcinoids, small cell and large cell neuroendocrine carcinomas. The authors discuss the genomic heterogeneity of pulmonary neuroendocrine tumors and the differences between low and high-grade neoplasms. Molecular signatures that do not necessarily follow the morphologic features could redefine the histological classification and provide better guidance for clinical management and tailored treatments for patients with pulmonary neuroendocrine tumors.

Sanja Dacic

dacics@upmc.edu

Philipp Ströbel

philipp.stroebel@med.uni-goettingen.de

1 Department of Pathology, University of Pittsburgh Medical Center, Pittsburgh, PA 15213, USA

2 Institute of Pathology, University Medical Center Göttingen, Robert-Koch-Str. 40, D-37075 Göttingen, Germany
Poorly differentiated and undifferentiated malignant neoplasms represent a diagnostically challenging group of tumors for thoracic pathologists. NUT carcinoma and thoracic SMARCA4 deficient undifferentiated tumors are examples of neoplasms that can only be diagnosed in the appropriate clinical settings and with the help of molecular techniques or specific immunohistochemical surrogate markers. The review of Chatzopoulos and Boland provides a detailed update on the historical relevance, clinico-pathologic features, genetics and differential diagnosis of these two unique thoracic tumors.

Immune-checkpoint inhibitor therapies have led to remarkable response rates and survival in a subset of patients with NSCLC. However, not all of the patients will show response to these therapies, and predictive biomarkers are still being investigated. Uruga and Mino-Kenudson review the pre-analytical, analytical, and post-analytical challenges associated with PD-L1 implementation in pathology laboratories. TMB has been extensively studied as an alternative or complementary biomarker of response to immune checkpoint inhibitors. The main issue with the assessment of TMB is the lack of a standardized approach. In addition, microsatellite instability (MSI) as a predictive biomarker in lung cancer is of limited value. Other potential biomarkers predicting the response to immune checkpoint inhibitors include immune cell profiling of the tumor microenvironment such as CD8+ T-cell density, IHN-g related mRNA profile, and the expression of MHC class I molecules, among others.

The treatment options for patients diagnosed with lung carcinomas have significantly changed in the past two decades. The development of new treatments is a rapidly evolving field and the identification of predictive biomarkers of response provides a personalized approach to the treatment of lung cancer patients. Canberk and Engels summarize testing approaches to the standard of care biomarkers such as EGFR, $A L K, R O S$ that are outlined in many national and international guidelines for molecular testing in lung cancer patients. Despite liquid biopsies paving their way into clinical practice, an adequate tissue sample remains the gold standard. Molecular laboratories are becoming more technically 
proficient in the use of cytology samples such as smears or pleural fluids for DNA and RNA assays.

In contrast to lung cancer, genomic studies in pleural mesotheliomas are limited. Molecular profiling of unique mesothelioma variants resulted in the revision of the histological classification as summarized in the review by Beasley MB and colleagues. For example, transcriptome analysis of transitional mesotheliomas, that were considered an epithelioid variant in the 2015 WHO classification, demonstrated unequivocal clustering with the sarcomatoid subtype supporting its reclassification as a cytological feature of a sarcomatoid mesothelioma. The authors have also provided a comprehensive review of a proposed concept of epithelioid mesothelioma architectural patterns, cytological and stromal features, and discuss a newly proposed prognostic two-tier grading system of epithelioid mesothelioma. Finally, the historically controversial concept of mesothelioma in situ is discussed in terms of clinical, morphologic, and molecular criteria required for the diagnosis.

Fernandez- Custa, Alcala and Foll offer an update on recently characterized transcriptome profiles of pleural mesotheliomas. Advanced bioinformatic approaches have shown strong inter-tumor molecular heterogeneity in a way that each mesothelioma can be considered to represent a combination of epithelioid-like and sarcomatoid-like components. Recent data shows that the prognosis of mesothelioma is best explained by a continuous model with the immune and vascular pathways being the major sources of molecular variation. These advances in our understanding of the molecular pathways may be relevant for development of new treatment options.

This annual review includes only a few topics related to non-neoplastic diseases of the lung. The use of electronic nicotine delivery systems has been very popular particularly among teenagers and reached epidemic proportions in the USA manifesting as acute and deadly respiratory disease. The Center for Disease Control and Prevention (CDC) termed this potentially fatal disease $E$ cigarette or vaping product use-associated lung injury (EVALI). In their review, Smith and colleagues discuss electronic nicotine delivery systems (ENDS) as well as the etiology, clinical presentation, imaging findings, pathologic features, treatment, and long-term consequences of EVALI. They conclude their article with a discussion of the impact of EVALI on the practice of pathology.

Over the last decade clinical, radiologic, and histopathological criteria have been established to define different entities of chronic diffuse parenchymal lung diseases. More recently, the clinical utility of this approach has been challenged. Verleden and colleagues discuss the molecular rationale for subtyping and prognostication of chronic fibrosing lung diseases by using blood, bronchoalveolar lavage, and tissue markers. As a purely molecular classification so far lacks sufficient sensitivity and specificity for subtyping, it is not yet routinely used and not implemented in international guidelines.
Alexander Marx and colleagues start the section on thymic tumors by reviewing the growing number of recurrent molecular alterations that have been described in thymomas in recent years and that have helped to underpin and confirm the classification approach used by the WHO. The Cancer Genome Project (TCGA) has shown that thymomas are among the adult tumors with the lowest mutational burden and only a few recurrent alterations. A rather intriguing finding in the years since is the fact that some thymomas show recurrent chromosomal translocations, such as the YAPMAML2 translocation in metaplastic thymoma. Potentially even more interesting is the recent description of KMT2AMAML translocations in some type B2 and B3 thymomas and occasional thymic carcinomas. However, as the authors point out, none of the molecular alterations described above does currently have therapeutic implications.

In their review on thymic carcinomas, Anja Roden and Malgrozata Szolkowska give a concise description of the epidemiological, clinical, and histopathological features of thymic carcinomas, a heterogeneous group of mediastinal cancers. Perhaps except for thymic squamous cell carcinomas (and the rare cases where a thymic carcinoma combines with a thymoma), most thymic carcinomas show few if any features that allow safe distinction from homologous carcinomas elsewhere in the body, making a close correlation with the clinical presentation mandatory. As the authors point out, most of the non-squamous cell types show the same key molecular alterations as in other organs, such as the $\mathrm{t}(11 ; 19)(\mathrm{q} 21 ; \mathrm{p} 13)(M E C T 1-M A M L 2)$ translocation in thymic mucoepidermoid carcinomas.

Neuroendocrine tumors of the thymus (TNET) are virtually indistinguishable especially from pulmonary neuroendocrine tumors, yet they show interesting epidemiological differences and there is increasing evidence that pulmonary and thymic carcinoids are different at the molecular level. Bohnenberger and Ströbel review recent data indicating that thymic neuroendocrine tumors contain a group of intermediate "NET G3" tumors. Moreover, this data shows that TNET fall into two major molecular groups, the low/intermediate grade group comprising typical and atypical carcinoids and the newly established "NET G3" on the one hand, and the high-grade group that consists of large cell and small cell neuroendocrine carcinomas.

Probably the most recent last-minute information comes from the review by Bösmüller and colleagues describing the pulmonary pathology of COVID-19. In their comprehensive article, the authors describe the histopathological pulmonary changes at different stages of the disease and summarize current knowledge about its pathophysiology. As the authors point out, COVID-19 induces mostly unspecific changes, perhaps with the important exception that early infections seem to be characterized by capillary microthrombosis, massive pulmonary edema, and epithelial damage, but little interstitial or 
alveolar inflammation, features that tell us much about the clinical manifestations of severe COVID-19 infections and that have also bearings for therapy. Importantly, much of this knowledge comes from multi-national autopsy programs and their coordinated tissue collections, highlighting once more the importance of a facet of clinical pathology that is considered negligible or even obsolete by some in the era of molecular sciences.
We hope you will find the selected topics interesting and informative. It has been our privilege to co-edit this Annual Review of Virchow's Archive.

Publisher's note Springer Nature remains neutral with regard to jurisdictional claims in published maps and institutional affiliations. 